\title{
Scanning electron microscopy of the development of structured aerial mycelia and satellite colonies of phenotypically switched Candida albicans
}

\author{
D. R. RADFORD, S. J. CHALLACOMBE* and J. D. WALTER \\ Departments of Prosthetic Dentistry and *Oral Medicine and Pathology, UMDS, Guy's Dental Hospital, \\ London SE1 9RT
}

\begin{abstract}
Candida albicans is an asexual diploid fungus that can express high frequency phenotypic switching. Switched variants can develop structured aerial mycelia (SAM) and cultures that have been grown for a protracted period exhibit satellite colonies. This study investigated the development of both SAM and satellite colonies by means of a freeze-drying technique and scanning electron microscopy. The results show that SAM may develop due to hyphae developing in the colony at the base of the structures. The development of satellite colonies is a result of cells that have grown deep into the agar re-emerging on to the agar/air surface and producing a new colony. Although both SAM and satellite colonies are often seen together on mature colonies, their cause and development are different.
\end{abstract}

\section{Introduction}

Although research for many years has been directed at Candida albicans as the cause of the most common forms of oral candidiasis (chronic atrophic candidiasis, chronic pseudomembranous candidiasis) there has been renewed interest as the organism is a major systemic pathogen in immunocompromised individuals. This interest has led to the 'rediscovery' of the phenomenon of phenotypic switching [1]. The potential of $C$. albicans to form variant colonies is well known [2]. Originally three main colony forms were described [3], although later a further 17 rough/hairy colony types were added of which five formed 'hairs', the morphological form 15 a producing the most prominent of these [4]. The term aerial hyphae was proposed in 1971 [5] although the first recorded reference to colonies being 'spiky and hard' was three decades earlier [3]. In this current report it is proposed to name these structures 'structured aerial mycelia' (SAM). This is because the term 'aerial hyphae' does not adequately describe these structures as they are not a single hypha but are composed of all three cell types. Furthermore, the adoption of the term SAM will avoid any possible confusion with true and pseudo hyphae. As a result of high frequency phenotypic switching

Received 3 July 1996; revised version accepted 10 Sept. 1996.

Corresponding author: Dr D. R. Radford. induced by ultraviolet radiation [1,6], additional types of colony such as 'ring' and 'hat' have been described recently.

High frequency phenotypic switching of $C$. albicans was first described in cultures of the type strain 3153A [1] grown on 'nutrient-poor agar' [7]. The agar contained amino acids, salts, vitamins and glucose and was produced to facilitate development of yeast and mycelial forms of $C$. albicans [8]. The general characteristics of switching in different strains are similar and include a basic 'o' smooth phenotype with reversibility and inter-convertibility between phenotypes. Switching can be induced by low dose ultraviolet irradiation with a limited number of predominant phenotypes [9].

Despite recent work on the DNA fingerprinting of phenotypically switched variant colonies, because of a belief that the phenomenon is mediated by genetic rearrangement $[7,10,11]$, few reports are available concerning the micro-structure of individual colonies [12-14]. Scanning electron microscopy (SEM) studies suggested that rough colonial variants were composed of a large number of pseudo-hyphal cells, although this was described for one colony only and little detail was presented [12]. Original work by Brown-Thomsen in 1968 [4] showed that variability of streaks of the organism was due to different proportions of blastospores, pseudo and true hyphae. More recent work related the cellular composition of six colony types of 
certain definite growth patterns of colonies and described in detail the composition of SAM [14].

The aim of this study was to investigate the structural composition of the development of SAM and the satellite colonies of mature ' $O$ ' smooth colonies by scanning electron microscopy and to relate development of characteristic colony morphology to patterns of dimorphic cell development during colony growth.

\section{Materials and methods}

\section{Culture technique}

The technique has been described previously [14]. Briefly, the identity of $C$. albicans NCPF $3153 \mathrm{~A}$ was confirmed by germ-tube formation in bovine serum and with the API 20C Aux system (bioMérieux, Basingstoke). A loopful of a single colony from Sabouraud agar was added to $10 \mathrm{ml}$ of sterile normal saline and dispersed with a vortex shaker. Cells were counted in a haemocytometer (modified Fuchs-Rosenthal) and the suspension was adjusted to give a final concentration of $1 \times 10^{3}$ cells $/ \mathrm{ml}$. Nutrient-poor agar without zinc supplement $[8,11]$ was inoculated with $100 \mu \mathrm{l}$ of suspension to give a density of $c .100$ cells/plate. The plates were incubated for 14 days at $25^{\circ} \mathrm{C}$ and representative plates were held for up to 12 weeks at $25^{\circ} \mathrm{C}$. Colonies whose morphology remained stable over this period were then selected for SEM examination and their biochemical characteristics were reconfirmed with API 20C Aux. Colonies were photographed with a SLR camera, 50-mm macro lens and monochrome film $(\times 10$ original magnification Kodak T-Max, Kodak Ltd, Hemel Hemstead).

\section{Preparation for scanning electron microscopy}

Samples of colonies and the nutrient agar were plunged into liquid nitrogen, frozen for $2 \mathrm{~min}$ and manually impact fractured with a scalpel blade in the centre of the air interface surface of a colony. Some colonies were observed to detach during freezing; however, the orientation of both the detached colony and its nutrient agar was readily interpreted. Individual fragments were placed for $8 \mathrm{~h}$ in an Edwards Pearce Tissue Drier (EPD3, Edwards High Vacuum International, Crawley). The samples were stored in a desiccator for not more than $48 \mathrm{~h}$ before being mounted on to aluminium stubs with silver colloidal paint (Agar Scientific Ltd, Stansted) and viewed by SEM (Hitachi S-520 Hitachi PLC, Wokingham) at $10 \mathrm{kV}$. Five colonies of each representative colony type were viewed and photographed (Kodak Plus-X 120 roll film, Kodak Ltd).

\section{Results}

\section{Colonies with SAM (fuzzy type)}

SAM were seen developing on colonies after growth for 2 weeks. The colonies were termed 'fuzzy type' due to the development of these structures and either had the overall appearance of ' $\mathrm{o}$ ' smooth or were slightly undulating. Fig. 1 shows a photograph of a 3-week-old colony with early development of SAM. At their base, mature SAM were up to $150 \mu \mathrm{m}$ thick, tapering to 6 $8 \mu \mathrm{m}$ at their extremities and reaching $600 \mu \mathrm{m}$ in length. The SAM were smooth, well ordered with compact surfaces (Figs. 2 and 3, field widths $180 \mu \mathrm{m}$ and $90 \mu \mathrm{m}$ ). Aberrant true hyphae at least $40 \mu \mathrm{m}$ long were seen occasionally between the bases of SAM; blastospores were seen on their surfaces (Fig. 2, field width $180 \mu \mathrm{m}$ ). Examination of more mature merged SAM revealed the evidence of true hyphae continuous at the junction of the merger (Fig. 4, field width $90 \mu \mathrm{m})$. The evidence of this series of photomicrographs as well as observations of other SAM (not illustrated) may indicate that true hyphae are the progenitors of merged SAM.

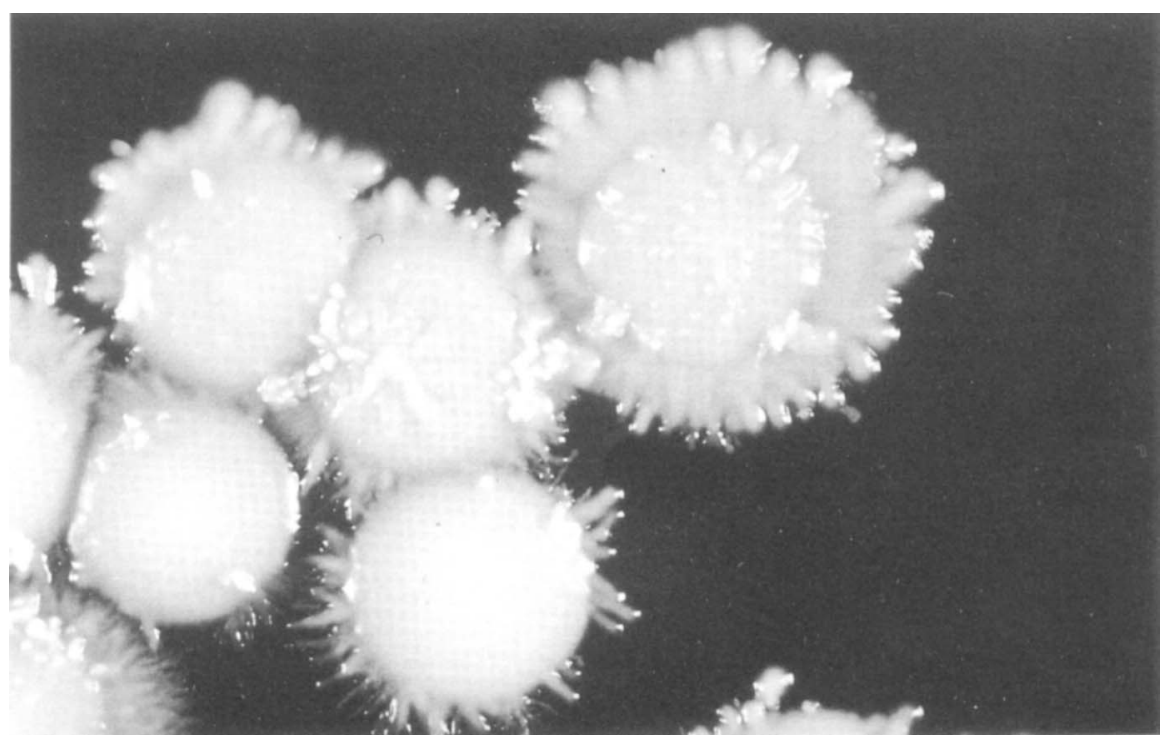

Fig. 1. The appearance of colonies showing early development of SAM (C. albicans $3153 \mathrm{~A})(\times 10)$. 


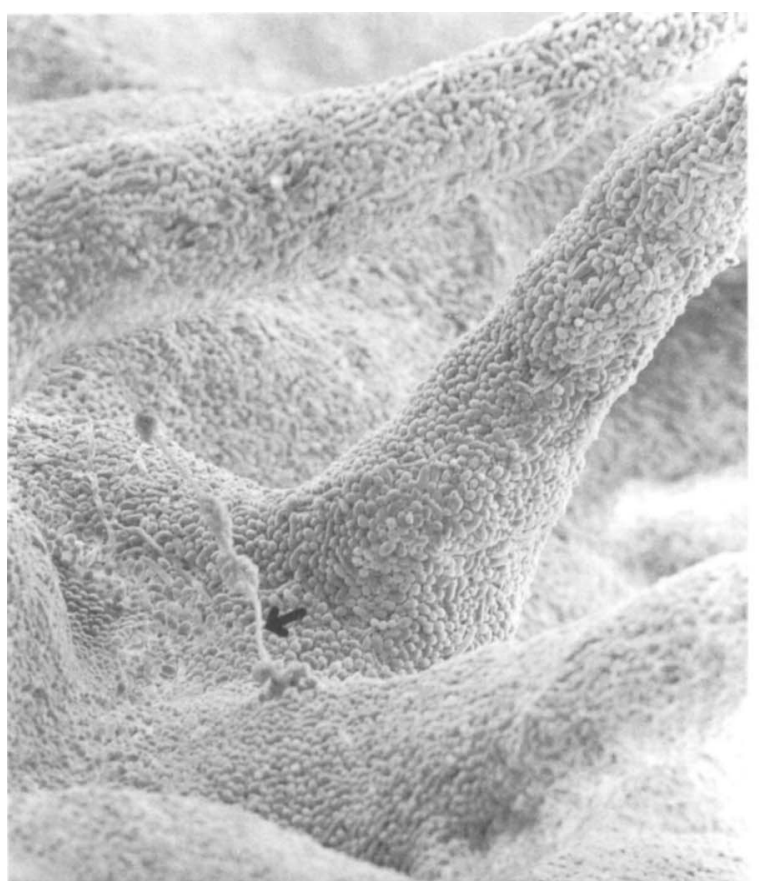

Fig. 2. Photomicrograph of the bases of mature SAM. Arrow indicates development of aberrant true hypha growing from one base to another $(\times 445)$.

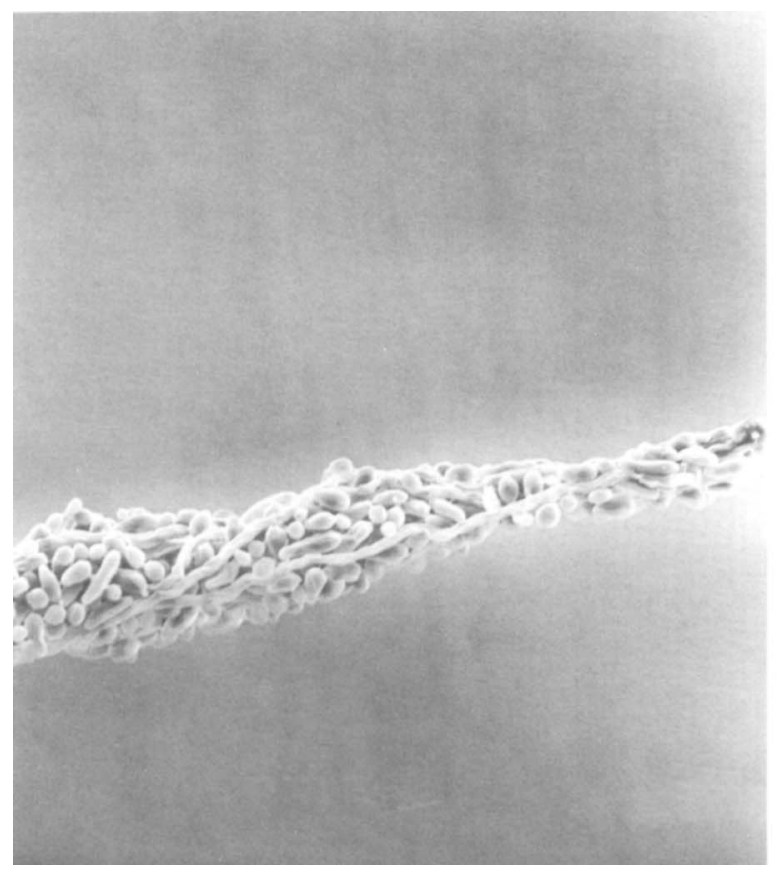

Fig. 3. Photomicrograph of the distal end of an aerial hypha showing compact nature and three cell types involved in growth $(\times 890)$.

The unusual formation of SAM led to examination of colonies that were just beginning to develop these structures. SAM either developed as a continuous ring around the centre of a colony or in discrete sites. They were short, stout structures initially (Fig. 5, field width $2.25 \mathrm{~mm}$ ) and as the colony matured the SAM

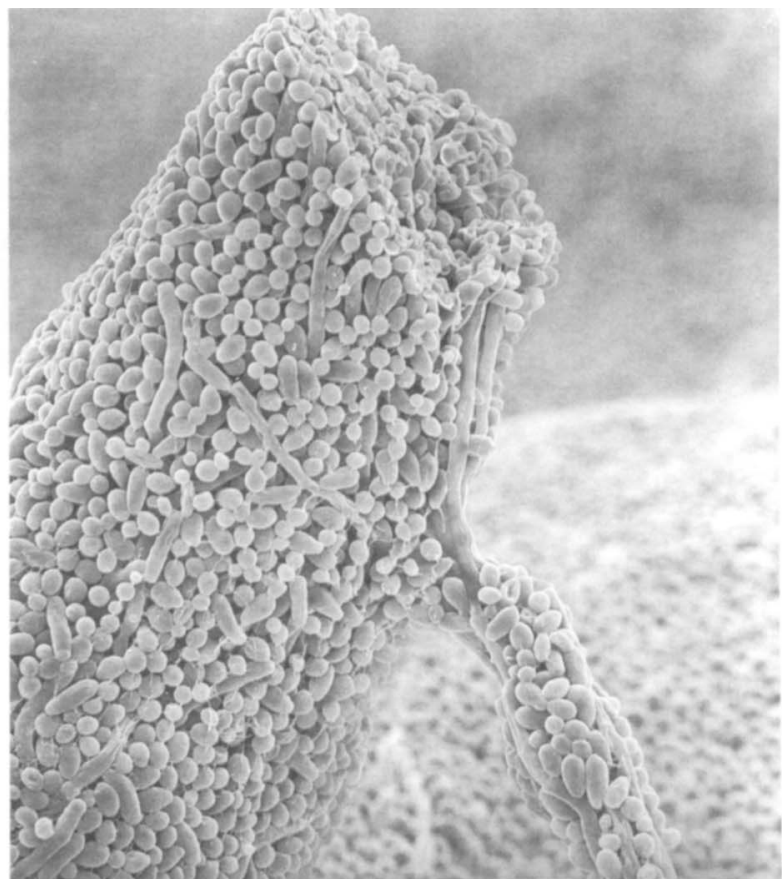

Fig. 4. Photomicrograph of mature merging SAM showing true hyphae at site of merger $(\times 880)$.

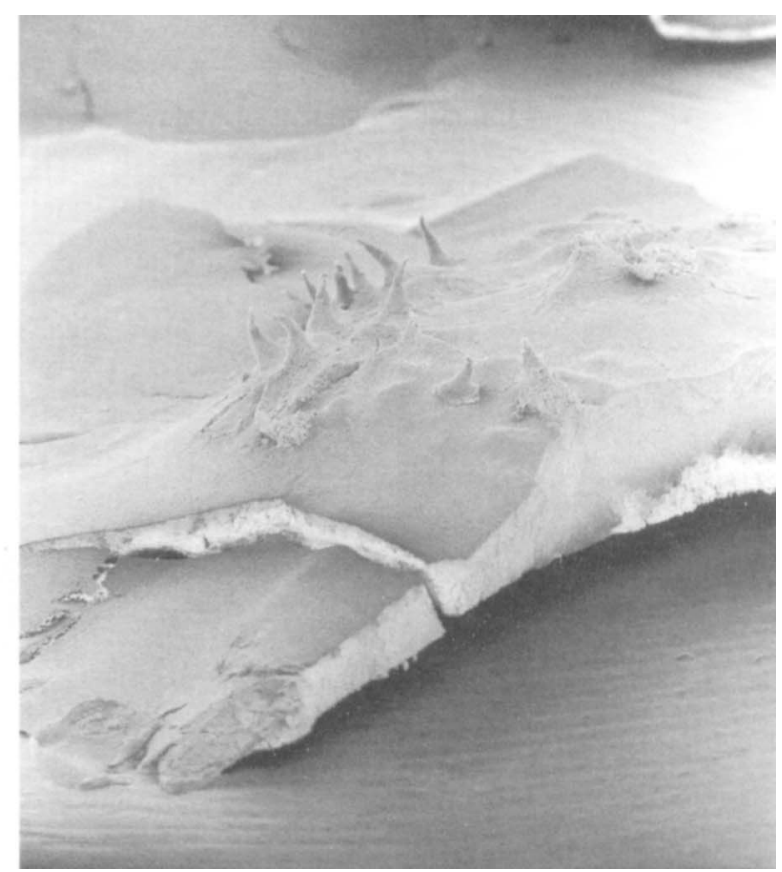

Fig. 5. Photomicrograph of a colony with immature SAM $(\times 35)$. Note that the hyphae are developing concurrently in a circle around the centre of the colony.

elongated. Fractured colonies allowed investigation of the longitudinal profile of these structures as well as through the immediately adjacent body of the colony. Immature SAM were composed of blastospores, true hyphae and pseudo-hyphae. Examination of the base of the structure and the adjacent area of the colony at 
high magnification showed the development of true hyphae and pseudo-hyphae (not illustrated).

\section{Mature 'o' smooth colonies showing satellite colonies}

To encourage switching, colonies were cultured both at a lower temperature and for considerably longer periods of time than is used for routine clinical diagnostic culture of yeasts. Therefore, many old cultures (up to 12 weeks) were observed in this study. These mature cultures yielded interesting observations, one of which was the development of satellite colonies around the edge of the main or mother colony (Fig. 6).

The SEM investigation of these colonies provided an explanation for the development of the satellite colonies. It was shown previously that colonies of $C$. albicans normally grew into agar that has a widely spaced honeycombed structure [14]. However, 10-12week-old agar was considerably more compacted due to drying of the material during the prolonged culture at $25^{\circ} \mathrm{C}$. Fig. $7 \mathrm{a}$ (field width $3.6 \mathrm{~mm}$ ) shows the nutrient agar mounted on the aluminium stub, the arrow indicates a nest of blastospores deep within the lower $25 \%$ of the agar (Fig. $7 \mathrm{~b}$ and c, field widths $900 \mu \mathrm{m}$ and $90 \mu \mathrm{m})$. Nests of colonies of blastospores with no apparent hyphal growth to account for their presence were observed deep in the agar. High magnification examination of the nests revealed the presence only of blastospores with evidence of budding at high magnification (not illustrated) with no hyphal formation (Fig. 7c, field width $90 \mu \mathrm{m}$ ).

Detachment of the colonies frequently occurred during freezing, although orientation of the separated speci- mens was easily interpreted. This permitted the surface of the agar and the surrounding area of a colony to be studied. Fig. 8a (field width $1.8 \mathrm{~mm}$ ) shows the superior surface of agar in the site of a detached mother colony. A small 'volcano' of blastospores that had emerged from the agar is arrowed. Other 'volcanoes' can be seen in the undulating zone of the agar which was at the periphery of a mother colony. The high magnification view (Fig. 8b, field width $90 \mu \mathrm{m}$ : orientation at $90^{\circ}$ to Fig. 8a) shows the agar which has split and blastospores have tumbled out on to the nutrient agar. At high magnification, budding cells were observed. These cells have the potential to form daughter or satellite colonies.

\section{Discussion}

C. albicans is an asexual diploid fungus that can develop four distinctive cellular morphologies: blastospores, true hyphae, pseudo-hyphae and chlamydospores. The first three of these morphologies are found in vivo. Chlamydospores are found only in vitro and are used as an identifying feature of $C$. albicans. Investigation of yeast infections in human tissues led to the observation that in deep-seated mycoses there was a mixture of budding yeasts, pseudo-hyphae and true hyphae [15]. C. albicans, like other microorganisms, is capable of phenotypic switching. Organisms that show this spontaneous switching resulting in antigenic variation are relatively common and include Salmonella Typhimurium and Neisseria gonorrhoeae. High frequency phenotypic switching occurs where a cell emanating from a single progenitor can express different phenotypes. This is characterised by variant

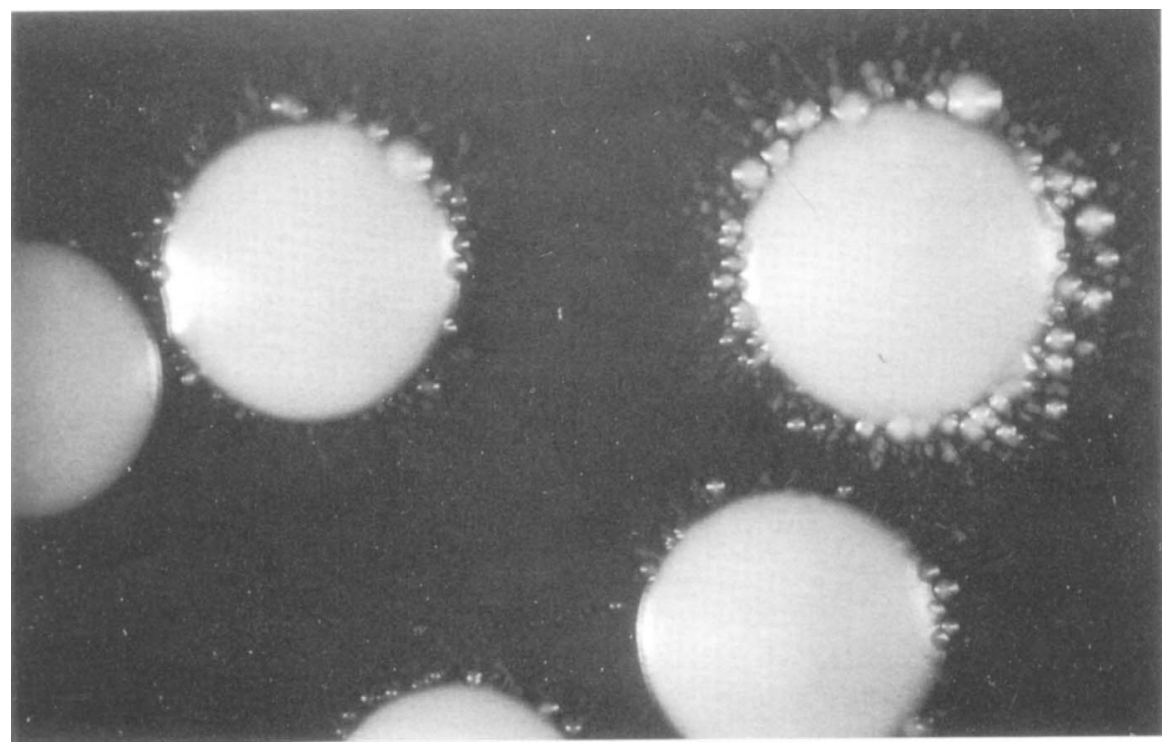

Fig. 6. Colonies grown over 12 weeks showing development of satellite colonies around the periphery (C. albicans $3153 \mathrm{~A})(\times 10)$. 

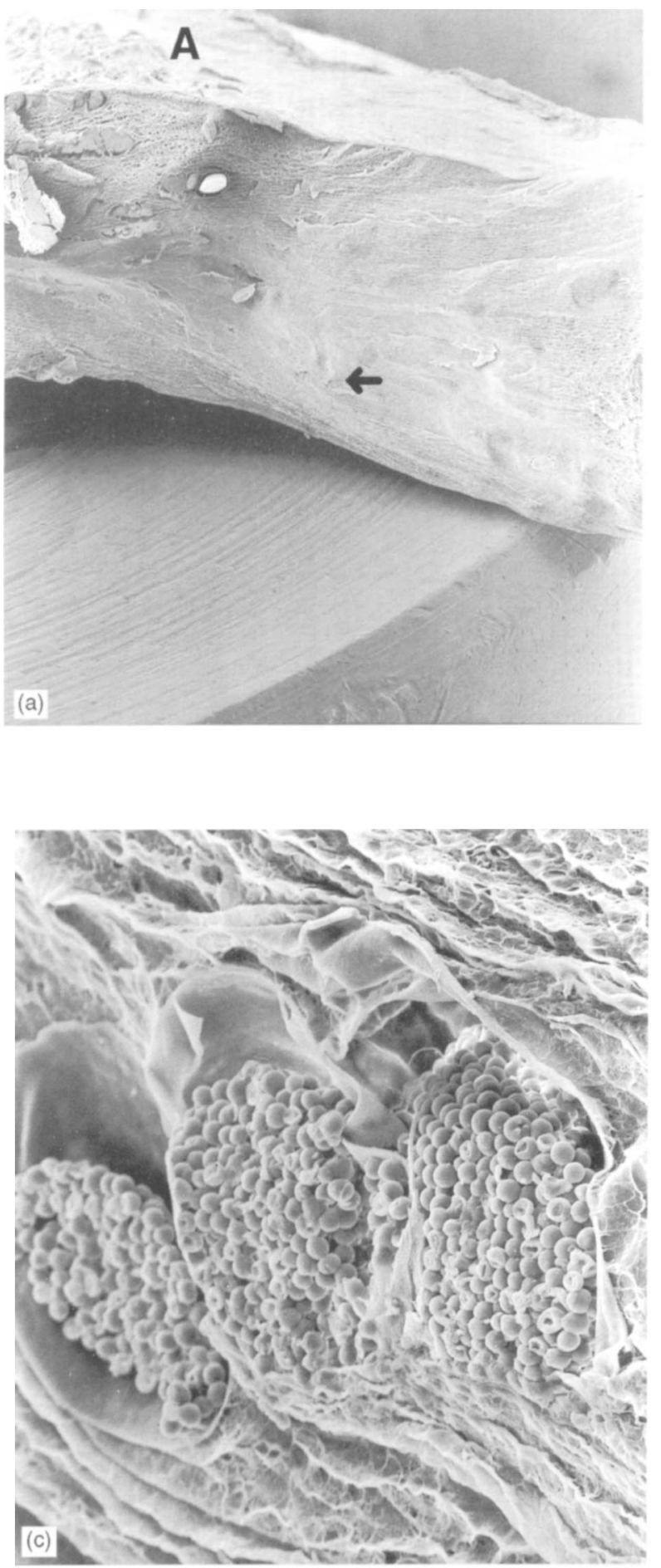

morphology of the organism when grown on nutrient agar [16].

In-vitro studies have concentrated on the different growth conditions that promote development of yeast and hyphal forms [8]. The appearance of smooth and rough colonies has also been reported $[2,4]$. Despite the interest in high frequency switching [1] and DNA fingerprinting of phenotypically switched variant colonies $[7,10]$, only limited work has been undertaken into the structural composition of variant colony

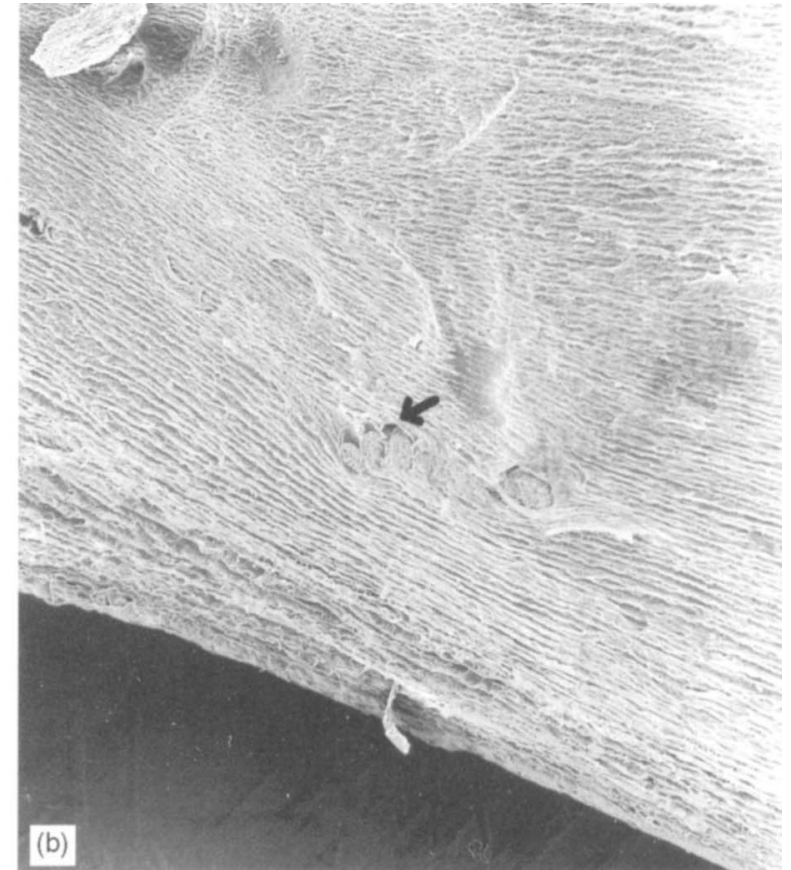

Fig. 7. a, Photomicrograph of mature nutrient agar (A) at the site of a detached colony $(\times 22)$. Arrow indicates nest of blastospores shown in $b$ and $\mathbf{c}$. $\mathbf{b}$, Photomicrograph of the triple nest of blastospores in the bottom $25 \%$ of the agar $(\times 90)$. c, Photomicrograph of the triple nest of blastospores in enlarged compartments of the agar with no evidence of hypha formation $(\times 890)$.

morphologies [13]. Studies with SEM suggested that rough colonial variants were composed of a large proportion of pseudo-hyphal cells rather than blastospores or true hyphae [12]. A later study with SEM and freeze-drying examined five commonly occurring variant colony types and 'o' smooth [14]. It was shown that colony morphology could be related directly to the cellular constituents of each type of colony. It was established that ' $\mathrm{o}$ ' smooth colonies consisted entirely of blastospores with other colony types being composed of blastospores and hyphae in 

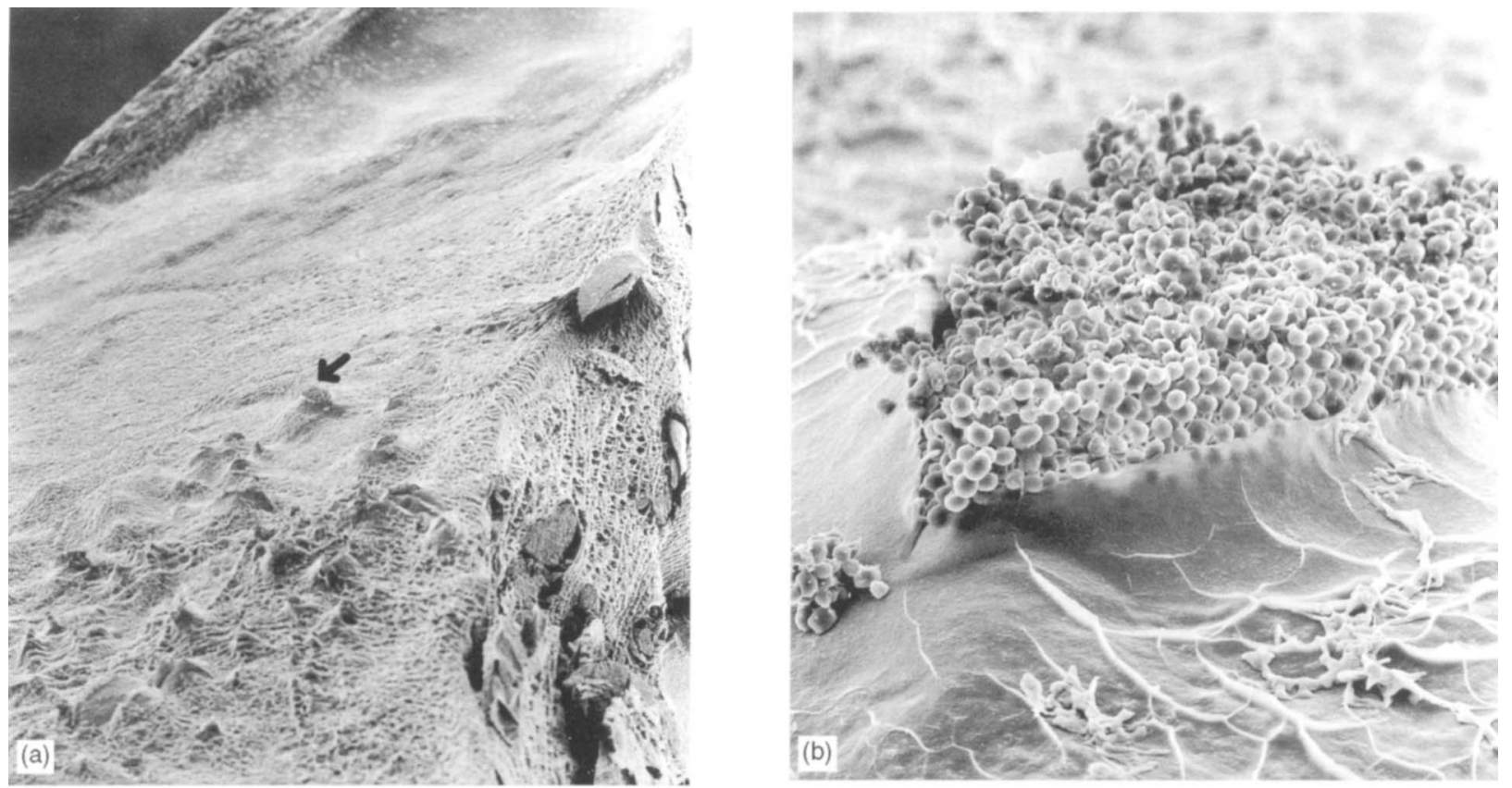

Fig. 8. a, Photomicrograph of the superior surface of the mature nutrient agar in the region of the satellite colonies $(\times 45)$. Arrow indicates a 'volcano' of blastospores. b, Photomicrograph of a 'volcano' of blastospores emerging on to the surface of the agar $(\times 890)$. Note the appearance of splitting of the surface caused by the budding blastospores.

different regions of the colony. This study was also the first to discuss and illustrate the cellular constituents of mature structured aerial mycelia [14].

Examination of the SAM might have been expected to indicate more clearly the stages in growth of agargrown $C$. albicans. If these structures had consisted of a defined core of hyphal cells with blastospores and pseudo-hyphal cells developing around that core, a pattern might have been discerned. However, no such pattern was found, instead an apparently haphazard collection of all three cell types was recorded. The development of such a structure remains difficult to understand, as daughter cells grow away from the nutrient source at a considerably faster rate than would have occurred if they had spread over the surface of the existing colony.

This study of the development of SAM clearly demonstrated that the colony grew at the air interface and not just close to the agar surface as had been described previously [13]. It is possible that there may have been some integration of the different forms of C. albicans to form such complex structures. There was some evidence in the mature hyphal development that merging of SAM may have occurred due to aberrant hyphae growing out of the otherwise smooth and well ordered surface of the structures. Blastospores were seen to have developed on these aberrant hyphae. The reasons for the development of SAM have not been discerned. Given their even spacing around the centre of immature colonies showing SAM development, this may have been an event caused by a stimulus of the phenotypically switched cells. There appeared to have been a concentration of true and pseudo-hyphal cells at the base of these unusual structures in one colony which may have accounted for their development. Previous work [5] has shown that aerial hyphae can be produced by $C$. albicans when cultured on a solid medium containing peptone, acid-hydrolysed casein, soluble starch and agar in an atmosphere of air with $\mathrm{CO}_{2} 10 \%$ both at $37^{\circ} \mathrm{C}$ and room temperature. With this medium some isolates started producing SAM from day 1 of culture, which differed from the present study where SAM were observed only after culture for 2 weeks.

The development of SAM illustrates the growth of the colony at the air interface; however, blastospores also penetrated deeply into the agar and reproduced asexually. When these 'nests' of cells or microcolonies occurred near to the surface of the agar, they had the ability to break through on to the surface due to the number and volume of the cells. This was not thought to be due to processing artefacts as the appearance of the split agar and emerging blastospores was seen only associated with the development of mother and satellite colonies. When on the surface of the nutrient agar they were able to form a new colony, thus giving rise to satellites. A further possibility of the appearance of the satellite colonies may be mechanical spattering due to microdroplet formation arising from handling disturbances during the long culture period. Care was taken at all times and the plates were incubated in a dedicated incubator for the duration of the culture period. 
In conclusion, SAM have a compact surface. Aberrant true hyphal growth between SAM probably accounts for the merging of these structures. The growth of SAM is possibly instigated by the local development of hyphae in the body of the colony. Satellite colonies are due to the downward and subsequent upward growth of blastospores in the body of the agar. These two cultural occurrences, although often seen together, have different origins. SAM are thought to be manifestations of phenotypic switching of C. albicans, whereas the development of satellite colonies is a simple growth phenomenon seen on mature agar plates.

We acknowledge the help of L. Fernandes of the Department of Oral Medicine and Pathology and K. Brady of the E.M. Unit, Guys campus, UMDS, for technical assistance.

\section{References}

1. Slutsky B, Buffo J, Soll DR. High-frequency switching of colony morphology in Candida albicans. Science 1985; 230: 666-669.

2. Di Menna ME. Natural occurrence of rough variant of a yeast, Candida albicans. Nature 1952; 169: 550-551.

3. Mackinnon JE. Dissociation in Candida albicans. J Infect Dis 1940; 66: $59-77$.

4. Brown-Thomsen J. Variability in Candida albicans. (Robin) Berkhout. I. Studies on morphology and biochemical activity. Hereditas 1968; 60: $355-398$.
5. Iralu V. Formation of aerial hyphae in Candida albicans. Appl Microbiol 1971; 22: 482-488.

6. Pomés R, Gil C, Nombela C. Genetic analysis of Candida albicans morphological mutants. $J$ Gen Microbiol 1985; 131: 2107-2113.

7. Anderson JM Soll DR. Unique phenotype of opaque cells in the white-opaque transition of Candida albicans. J Bacteriol 1987; 169: $5579-5588$.

8. Lee KL, Buckley HR, Campbell CC. An amino acid liquid synthetic medium for the development of mycelial and yeast forms of Candida albicans. Sabouraudia 1975; 13: 148-153.

9. Soll DR, Morrow B, Srikantha $T$, Vargas W, Wertz P. Developmental and molecular biology of switching in Candida albicans. Oral Surg Oral Med Oral Pathol 1994; 78: 194-201.

10. Suzuki T, Kobayashi I, Kanbe T, Tanaka K. High frequency variation of colony morphology and chromosome reorganization in the pathogenic yeast Candida albicans. $J$ Gen Microbiol 1989; 135: 425-434.

11. Rustchenko-Bulgac EP, Sherman F, Hicks JB. Chromosomal rearrangements associated with morphological mutants provide a means for genetic variation of Candida albicans. $J$ Bacteriol 1990; 172: $1276-1283$.

12. Joshi KR, Gavin JB. The morphology of colony variants of three species of Candida. Sabouraudia 1975; 13: 274-279.

13. Whittaker DK, Drucker DB. Scanning electron microscopy of intact colonies of microorganisms. J Bacteriol 1970; 104: 902-909.

14. Radford DR, Challacombe SJ, Walter JD. A scanning electronmicroscopy investigation of the structure of colonies of different morphologies produced by phenotypic switching of Candida albicans. J Med Microbiol 1994; 40: 416-423.

15. Salfelder K, Ueda K, Quiroga EL, Schwarz J. Visceral candidosis. Anatomic study of 34 cases. Curr Top Pathol 1977; 64: 177-224.

16. Soll DR. High-frequency switching in Candida albicans. Clin Microbiol Rev 1992; 5: 183-203. 